\title{
NOUVELLES QUESTIONS FÉMINISTES: 22 AÑOS PROFUNDIZANDO EN UNA VISIÓN FEMINISTA, RADICAL, MATERIALISTA Y ANTI-ESENCIALISTA
}

\author{
JULES FALQUET \\ Université de Paris VII - Jussieu
}

\begin{abstract}
Resumen: Este artículo presenta las grandes orientaciones teóricas y políticas, así como la história, de la más antigua revista científica feminista francófona Nouvelles Questions Féministes, que fue fundada hace 22 años por Simone de Beauvoir, entre otras. Esta revista se caracteriza por su voluntad de unir calidad teórica y anclaje político y militante, en una perspectiva radical, materialista y anti-esencialista muy firme. A lo largo de su existencia, Nouvelles Questions Féministes ha publicado artículos de especial importancia para el movimiento feminista. A mediados de los difíciles años 90 , empieza a tener serias dificultades para mantenerse, sin apoyo institucional, hasta casi cerrar. Sin embargo, ha empezado una nueva vida a partir de 2001, cuando es retomada - con la misma perspectiva política - por un dinámico grupo alrededor de la Universidad de Lausanne.
\end{abstract}

Palabras-claves: feminista, revista, materialista, radical, anti-esencialista

Con sus 22 años de existencia, Nouvelles Questions Féministes es la revista feminista francófona más antigua que existe hoy día. Su historia, que aquí esbozaré, coincide con la historia del desarrollo y transformación del movimiento feminista francés, en el que ocupa un lugar importante, tanto como herramienta para la profundización teórica de numerosas militantes, investigadoras y docentes francófonas, como por constituir un espacio para la expresión del mismo movimiento. Dentro del paisaje de las revistas feministas francófonas actuales, NQF tiene una doble especificidad: primero, su voluntad de unir calidad teórica y perspectiva política feminista, militante. Segundo, su promoción de una línea de análisis claramente materialista, radical y anti-esencialista.

Aquí presentaré algunos elementos históricos y analíticos de reflexión sobre $N Q F$, a la cual contribuyo desde mediados de 1995. Obviamente, aunque intente reflejar lo mejor posible las orientaciones comunes del comité de redacción, también externaré aquí mis análisis y convicciones personales. Primero les presentaré un resumen de la historia de la revista en Paris, luego enfocaré las perspectivas teórico-políticas y el funcionamiento práctico de $N Q F$ en el periodo actual, desde su renacimiento en Lausanne. Terminaré con un balance de nuestros 22 largos años de hacer feminismo desde la publicación de una revista (entre otros medios) y con nuestros proyectos actuales. 


\section{Algunos elementos históricos}

Antes que nada, es preciso recordar que existe una prehistoria de $N Q F$. Y es la revista Questions Féministes, fundada en 1977 en la época de auge del movimiento feminista francés, por Simone de Beauvoir, Christine Delphy, Colette Capitan-Peter, Emmanuelle de Lesseps, Nicole-Claude Mathieu y Monique Plaza. Se trata de la primera revista de este tipo, o sea: existe en estos años una prensa feminista floreciente, pero las revistas son más de opinión y de combate, con textos breves, de humor e informativos. Sobre todo, lo que publica cada una es la expresión del grupo que lo redacta -su foro. Aparecen y desaparecen a medida que los colectivos se deshacen. $Q F$, en cambio, se orienta desde el principio hacia artículos largos, "de fondo", y sobre todo, está hecha para la expresión de todas. Si bien las miembras del colectivo de la revista escriben en ella, $Q F$ busca publicar textos de otras feministas, traducciones, en fin: abrir un verdadero espacio de debate político y teórico.

En aquella época, al fragor de la lucha, se publican muchos artículos claves para la reflexión feminista, entre los cuales vale la pena mencionar los dos artículos maestros de Monique Wittig: "No se nace mujer" y "El pensamiento straight". La revista abre muchos debates, introduce autoras extranjeras y problemáticas: realiza un trabajo verdaderamente pionero. Sin embargo, a raíz de cruentos debates y profundos desacuerdos políticos sobre la cuestión del lesbianismo radical, el grupo inicial de $Q F$ se divide durante el verano 1980 , al tiempo que emerge un movimiento lésbico con fuertes cuestionamientos al "heterofeminismo", que el movimiento feminista francés conoce una primera fase de transformación con la consecución de la despenalización del aborto, y que llega al poder un gobierno socialista que mucho ha prometido a las mujeres. De este primer periodo quedan ocho números de una revista muy densa, atesorados por bibliotecas y coleccionistas, que sentaron las bases del análisis feminista radical y materialista francés, enraizado tanto en la reflexión teórica como en la lucha militante.

\section{1. Los años 80: acompañando el movimiento feminista y el desarrollo de los estudios del mismo nombre}

En 1981, Simone de Beauvoir, Christine Delphy, Claude Hennequin y Emmanuèle de Lesseps vuelven a juntarse para formar la revista de la cual ahora hablaré: NQF. En el editorial del primer número reafirman que sus orientaciones políticas no han cambiado y que mantienen su "perspectiva radical" (lo que en Francia significa que no son ni diferencialistas, ni de la tendencia "lucha de clase", sino materialistas y autónomas). Recalcan de nuevo su voluntad de ser un espacio de debate teórico-político para el movimiento y, también, de dar a conocer textos de mujeres no francófonas, poniéndose como objetivo publicar al menos una traducción en cada número. Repitámoslo: la especificidad de la revista es su doble anclaje, en el movimiento y en una perspectiva científico-teórica. NQF es la precursora, en Francia, de las revistas feministas universitarias que surgen varios años después. Incluso, cuando se organiza el primer gran coloquio científico en Toulouse en 1982, "Mujeres, feminismo e investigación", apoyado por el CNRS (Centre National de la Récherche Scientifique), además de las docentes que valientemente han introducido el tema del feminismo en sus clases, la existencia de $N Q F$ y su contenido permiten comprobar que realmente existe un campo científico feminista vigoroso e innovador.

Al principio, como buena revista movimentista, $N Q F$ tiene una aparición un tanto irregular de más o menos un número anual. Cada número se organiza alrededor de un tema central, entre los cuales destacan: la importancia y validez de construir un "feminismo materialista" ( $\left.n^{\circ} 4\right)$, "Ias mujeres y el Estado" ( $\left.n^{\circ} 6-7\right)$, un especial en contra de la esclavitud 
sexual ( $n^{\circ} 8$ ), un número sobre la situación de las mujeres de las Antillas ( $n^{\circ}$ 9-10), y otro sobre "la militarización y la violencia hacia las mujeres" ( $\left.n^{\circ} 11-12\right)$. Navega entre la formalidad y la informalidad: hay un comité de redacción pero se menciona en media página, donde haya espacio. Las rúbricas son variadas e incluyen un apartado grande para dar cuenta de acciones del movimiento y de voces "de la base", provenientes de dentro y fuera del país (gracias a las traductoras benévolas), así como para tomar posición en los debates político-científicos del momento.

En este sentido, merece una mención especial el $n^{\circ} 5$ de 1985 sobre "el patriarcado en el campo/en campaña", no sólo por su apertura a problemáticas más allá de Europa, con contribuciones sobre Tunicia y América Latina, sino también por un texto breve titulado "Crónicas de una impostura". Este texto denuncia el "trabajo" del famoso sociólogo francés de los movimientos sociales, Alain Touraine, sobre el movimiento de mujeres. Las denunciantes, ocho mujeres que han participado en uno de los grupos "investigado" por Touraine, exponen las profundas debilidades de su metodología y subrayan su "deshonestidad intelectual". De hecho, Touraine nunca se atrevió a publicar los resultados de esta investigación, en la que concluía que el movimiento de liberación de las mujeres no era un movimiento sino una secta. Aunque Touraine se haya ganado la fama de un prominente teórico de los "nuevos movimientos sociales" dentro de la sociología dominante, queda registrada públicamente, gracias a $N Q F$, la debilidad de su construcción científica. Nostalgia de una época en la que las feministas, tanto desde el movimiento como desde la teoría y la academia, sentían la libertad y la audacia suficientes para abrir debates verdaderamente críticos con el pensamiento dominante.

Después de cinco años álgidos, de mucho trabajo, debates abiertos y aportes teóricos, $N Q F$ tiene un primer período de interrupción en 1986, por falta de dinero. Sin embargo, vuelve a salir de nuevo en 1991 con un número triple especialmente nutrido sobre "particularismo y universalismo". Es revelador leer el editorial de Christine Delphy, para entender hasta qué punto la revista está imbricada en el contexto tanto del movimiento -debilitado-, como en el de los estudios feministas, que se han empezado a visibilizar y desarrollar desde el "coloquio fundador" de Toulouse en 1982, pero están quedando de nuevo en la invisibilidad. Ridiculizando con mucho brío y rabia la "excepción francesa", "la famosa risa gala -y científica" que tienen que enfrentar todavía los estudios feministas en el país, haciendo una crítica sin concesión de la política del gobierno socialista, y luego de derecha, Delphy explica su larga e infructuosa búsqueda de financiamiento para la revista. Pero también subraya las debilidades de las propias investigadoras feministas, su falta de organización e incluso de solidaridad. Es cruel, pero bastante profunda y estimulante, su denuncia del "consumismo" de muchas investigadoras y docentes en Francia de cara a las revistas feministas - querían que existieran, pero ni siquiera se suscribían. El hecho de sacar este $N Q F$ después de varios años de interrupción, búsqueda de subvenciones y desilusiones -iun número triple, además!- es una manera de burlarse de tanta inercia y de comprobar que el feminismo tiene mucho que decir. Pero, ¿habrá otro número? ¿Seguirán los estudios feministas, y cómo? NQF se ubica decididamente en la lucha, con el movimiento: "[hace falta una voluntad política] Son las interesadas - investigadoras, docentes, estudiantes - quienes deben suscitarla: ¿tendrán ellas la fuerza de hacerlo sin el apoyo de todas las feministas? Finalmente, es a todas las feministas de este país a quienes les toca preguntarse si quieren estudios feministas. Si los quieren, tendrán que pelear por ellos, mucho. iComo siempre!"1

La presentación que a continuación hace Françoise Armengaud del contenido de este NQF 16-17-18 completa el tono de lucha y manifiesta la inserción de la revista en la actualidad internacional -la lucha de las mujeres en Argelia ("NQF no tiene otro objetivo que no sea servir de expresión y de instrumento a la lucha de las mujeres"2)- y en la 
actualidad de los debates teórico- políticos -el combate al diferencialismo-naturalista de Irigaray y de la "reivindicación maternalista", la promoción de un punto de vista lésbico, pero no como una "diferencia [.] ni un particularismo, [sino como] un revelador y algo que nos empuja hacía el universalismo" 3 . Los demás temas (la energía nuclear en contra de la democracia, el trabajo doméstico femenino visto a través del ejemplo de las brujas, un texto de información sobre el movimiento feminista en Brasil escrito por Andrée Michel) ilustran la forma en que se unen las múltiples raíces de la revista en una propuesta políticoteórica única y bastante alentadora.

\section{2. Los 90: resistiendo el backlash}

Afortunadamente, la incierta apuesta de 1991 se concretiza: a partir de 1992, NQF cuenta por fin con algunas subvenciones. Cambia de portada, de formato; formaliza su aparición con cuatro números al año. Se ha domiciliado en una sede académica, en un instituto de investigación muy reconocido, el IRESCO (Instituto de Investigación y de Estudio sobre las Sociedades Contemporáneas). Está lista para un nuevo comienzo. Lo que hace falta, como lo diagnosticó el editorial de 1991, es iel movimiento! Después de muchos años de social-democracia al poder, de crisis y de desempleo, se ha vuelto la sombra de lo que fue. Tal como lo describe Susan Faludi, el backlash ha empezado: el retroceso social, ideológico y político se hace sentir con más fuerza a medida que avanza el decenio.

A pesar del contexto adverso, $N Q F$ saca problemáticas de fondo y publica sobre "nacionalismo y anti-feminismo", el conflicto entre Israel y Palestina, el movimiento en Turquía, las violaciones en ex-Yugoslavia, las mujeres del mundo mediterráneo, pero también, sobre mujeres y filosofía, las mujeres en lo político, el lugar de las mujeres en el mundo científico. Gracias a la tenacidad de las redactoras y a su complicidad, a puro pulmón, la revista sigue ocupando valientemente el lugar que ha escogido.

Mi encuentro personal con NQF empieza en 1995, cuando entro a favor de una mini-renovación del Comité de redacción, que aporta al equipo un poco de las tan ansiadas energías nuevas: iapenas somos cinco! Sin embargo, la revista sigue saliendo lo más cabalmente que se puede -4 números al año implican un ritmo de trabajo aplastante. Es un reto alimentar a $N Q F$ en estos años en que todavía no despuntan las nuevas generaciones de investigadoras feministas en Francia y en el mundo francófono, iy en que no se tiene dinero para traducir los tantos textos que necesitarían conocer las francófonas! Porque claro: en el resto del mundo, el feminismo avanza -la impresión de estancamiento que se siente en Francia es aún más difícil de soportar. Entre subvenciones y suscripciones, apenas se malcubre un secretariado de redacción a tiempo muy parcial y algunos envíos solidarios de la revista: la impresión, por su parte, muchas veces se paga con dinero prestado.

Temas para hablar y analizar, hay muchos. En ese periodo, la revista ofrece dos números importantes sobre el esencialismo-naturalismo, dos sobre el lesbianismo y la heterosexualidad, y otros dos sobre la violencia contra las mujeres. Cuando se empieza a hablar de la "paridad", y a manejar que la democracia debería de ser, en cada puesto de poder, "una mujer, un hombre", las opiniones en el movimiento feminista son bastante diversas. NQF es la única revista que se propone participar abiertamente en el debate, o mejor dicho, que abre un espacio para profundizar sobre la cuestión, que no es tan simple como parece. Tan encontradas son las posiciones que NQF decide publicar dos números: uno a favor de la paridad, otro en contra (respectivamente el Vol. 15, n 4 de 94 y el Vol. 16, $n^{\circ} 2$ de 95). Gracias a la presencia de una hispanófona en el Comité de redacción, $N Q F$ también empieza a abrirse hacía la producción teórica y los movimientos de mujeres y feministas latinoamericanas y del Caribe, primero publicando varios artículos, 
manifiestos, y documentos sobre la región, y luego sacando un número especial sobre feminismos en la región (Vol. 20, n³, 1999). Entre las autoras de estos textos, no sólo se encuentran investigadoras francófonas, sino también autoras latinoamericanas y caribeñas -aunque las traducciones se hagan fundamentalmente de forma voluntaria, militante: la falta de dinero y recursos siempre obliga a la revista a limitar sus ambiciones.

Sin embargo, pese a estos esfuerzos y emprendimientos, el comité de redacción está exhausto y los recursos van menguando a tal grado que, en 1999, el comité está a punto de tirar la toalla. Se decide finalmente, con el alma en un hilo, suspender la publicación, aunque sin clausurar definitivamente la revista. Afortunadamente, por una de estas felices coincidencias del feminismo internacional, a los pocos meses Christine Delphy, la responsable de publicación y alma de $N Q F$, viaja invitada a Suiza por varios colectivos feministas a dar una conferencia sobre el aborto y a intervenir sobre el patriarcado en la "Maestría en estudios de género" de la universidad de Ginebra, invitada por Patricia Roux. Gracias al entusiasmo y la organización de las feministas suizas, su viaje resulta en tres días de intensos debates; se traban complicidades y nace el proyecto de retomar NQF por medio de una colaboración franco-suiza.

\section{3. Entrando al nuevo milenio atajando por la academia suiza}

Para entender la nueva etapa de $N Q F$, hay que recordar algunos elementos de la historia del feminismo en Suiza. Allá, la década de los noventa empieza con una huelga nacional del trabajo doméstico de las mujeres, el 14 de junio 1991, que marca el renacimiento de varios colectivos y cierta radicalización del discurso feminista. Simultáneamente, los estudios feministas empiezan a salir de la semi-clandestinidad en la que se hallaban: en 1995, la universidad de Ginebra abre una Maestría en estudios de género, que luego se coordina con la universidad de Lausanne. En el 2000, en esta misma universidad de Lausanne, se consigue abrir un puesto de profesora en estudios de género -después de una huelga estudiantil que reivindicaba, entre otras cosas, un puesto en estudios feministas. En 2002, finalmente, se abre un puesto similar en la universidad de Bâle, y a los pocos meses aparece una escuela doctoral en estudios de género.

Este proceso de institucionalización de los estudios feministas bajo el nombre de "género" también se manifiesta a través de la creación de redes que permiten visibilizar el tema. La red LIEGE (Laboratorio Interuniversitario en Estudios de Género), creada en 2001 en Lausanne, juega un papel central en la nueva etapa de NQF. Goza de subvenciones oficiales y en apenas dos años ha logrado aglutinar a más de doscientos participantes en Suiza sobre todo francófona. Tiene una estructura horizontal, integrando estudiantes, doctorandos, investigadoras/es, y profesoras/es que se ubican en una perspectiva científica feminista. Es fundamentalmente gracias al LIEGE que NQF ha podido revivir: unas treinta de sus miembras se implicaron con entusiasmo en el nuevo Comité de redacción de la revista, y la coordinación de la red se encarga de todo el trabajo de secretariado de redacción de la revista.

\section{Manteniendo y profundizando la orientación claramente feminista, radical, materialista y esencialista}

\section{1. La difícil fidelidad a una perspectiva radical.}

Revista nueva o fiel continuadora de NQF-Paris: NQF tenía que aclarar las condiciones de su reaparición. Para tales fines, se decidió realizar un proceso colectivo de discusión para redactar una plataforma de la revista, cuya línea original había sido explicitada por última vez en 1977 en el primer editorial de $Q F$, y reafirmada en el primer editorial de NQF en 
1981. Dado que el Comité se amplió considerablemente, los debates resultaron largos y bastante ricos. Sin embargo, de forma muy significativa, no fue tan fácil ponerse de acuerdo sobre la necesidad de seguir reivindicando los conceptos de "radicalidad" y de "materialismo". Aunque todas estuvieran convencidas de la importancia de esta herencia teórico-política, afloraron las dudas a la hora de plasmar tan "pesadas" palabras en nuestra plataforma, en una época en que, por fin, aparece para el feminismo -o más bien para la perspectiva de género- la posibilidad de alcanzar una real legitimidad científica.

De hecho, tanto en Francia como en Suiza, las nuevas normas europeas e internacionales a favor de la "equidad de género" (égalité des chances) han contribuido al desarrollo y creciente visibilidad de una nueva -aún modesta- ola de "feminismo académico". En los últimos años se han formado asociaciones y redes de estudiantes, investigadoras o docentes en género y feministas; se han creado algunas plazas de docencia e investigación; se han multiplicado los eventos científicos; y se han creado o consolidado algunas revistas alusivas al tema. Pero precisamente, a la vez que se da y se busca esta legitimación, los conceptos evolucionan sutilmente y se observa un progresivo desplazamiento de apelaciones y perspectivas explícitamente feministas para perspectivas de género. Si bien es cierto que la teoría sexo-género puede representar un avance en determinadas disciplinas y lugares, también puede significar un retroceso por su aspecto más light, y la falta de claridad con que a menudo se maneja. Es en este contexto que el debate sobre el uso de la palabra "radical" toma todo su significado.

$N Q F$ se encontró entonces muy concretamente frente a un profundo interrogante: ¿era necesario "normalizar" su vocabulario para seguir consolidando su legitimidad académica (en un momento en que por primera vez está claramente vinculada a un proyecto universitario, e integra a muchas estudiantes y doctorandas que tienen que pensar en su porvenir académico), o debía seguir construyendo esta legitimidad -teórica, social y académica- sin renunciar a nada de su especificidad radical, militante y política? Sabiamente, optó por combinar los dos aspectos, convencida de que su posición específica sigue siendo necesaria en el panorama editorial y político francófono. Así, habiendo reafirmado su anclaje académico (en la composición del equipo, en los lugares de reunión, en el funcionamiento práctico), en lo que a su línea política se refiere, optó por reivindicar claramente una posición radical, materialista y anti-esencialista. Y de hecho, pensamos que: (1) hace falta un espacio de publicación específico para ciertos trabajos teóricos que no tienen realmente cabida en las revistas "de género" y académicas, porque van más allá de ellas y cuestionan hasta los criterios de legitimidad científica dominantes; (2) hace falta un polo teórico-político feminista fuerte, que no corra el riesgo de deslizarse hacia posiciones solapadamente esencialistas, idealistas y reformistas, a las que el backlash ideológico nos empuja constantemente.

\section{2. ¿Qué entendemos por feminista, radical, materialista y anti- esencialista?}

Como bien lo manifiesta la Plataforma de $N Q F$, resultado de las discusiones recién mencionadas y publicadas en 2003 , la revista se dedica a apoyar el desarrollo y la difusión de reflexiones teóricas y políticas enraizadas en el movimiento y las acciones feministas, desde la posición crítica que siempre ha constituido su originalidad. $N Q F$ se niega a explicar la subordinación y discriminación de las mujeres por parte de los hombres, por la naturaleza o por la biología. Desde nuestra perspectiva materialista y anti-esencialista, "mujeres" y "hombres" no son otra cosa que dos categorías sociales, producidas por y dentro de relaciones de poder organizadas en un sistema social que se llama patriarcado. A pesar de todos los actuales discursos sobre la "igualdad" entre los sexos, las posiciones sociales de 
las mujeres y de los hombres siguen siendo jerarquizadas, y siguen obligando a las mujeres a estar en relaciones de dependencia material y simbólica con respecto a los hombres.

Hemos mantenido una perspectiva radical en el sentido en que el horizonte de $N Q F$ es la transformación radical de las relaciones sociales de sexo y no un reacomodamiento de algunos elementos. Para tales fines, NQF busca seguir desconstruyendo la división y la supuesta diferencia entre los sexos que estructuran el conjunto de las relaciones sociales, y que no es más que una arbitrariedad orientada a legitimar el orden patriarcal. Al ser múltiples las opresiones que viven las mujeres, NQF quiere también estar abierta a todos los análisis que articulan la dimensión de sexo con otros mecanismos de categorización, jerarquización y opresión que organizan las relaciones sociales -en especial la clase, la "raza", la etnia y la sexualidad. Para tales fines, la revista publica artículos y testimonios provenientes de las sociedades occidentales, pero también de otras sociedades y regiones del mundo.

\section{3. El nuevo equipo y los métodos de trabajo}

Con más de 50 participantes, el nuevo Comité de redacción constituye probablemente uno de los más numerosos de la historia de las revistas feministas. Se reúne en la Universidad de Lausanne y sigue participando el grupo de Paris (menos una miembra, por razones de sobrecarga de trabajo). De hecho la revista está bajo la doble responsabilidad de Patricia Roux, de Lausanne, y Christine Delphy, de Paris. El Comité ha rejuvenecido considerablemente: ahora las treinteañeras son mayoría, y conjuga experiencias múltiples. En cuanto a disciplinas científicas, aunque sigue teniendo un fuerte anclaje sociológico, se ha abierto a un sinfín de otras disciplinas (literatura, ciencias políticas, historia, antropología, filosofía, derecho). En cuanto a las formas de compromiso político, el comité se enorgullece de contar con militantes a la vez que académicas, y varias mujeres que combinan la militancia con su trabajo académico. En resumidas cuentas, en esta nueva fase de la vida de $N Q F$, el anclaje académico de la revista se ha hecho mucho más evidente y sólido, y su nueva vitalidad es manifiesta. Vale la pena recordar que el comité de redacción sólo incluye mujeres.

El papel del Comité de redacción, que tiene tres reuniones ordinarias al año (después de la salida de cada número, para poder hacer un balance del mismo), se centra en definir las temáticas de los números de la revista, sus orientaciones científicas y políticas. Para el trabajo "efectivo" de realización de cada número, se forman grupos de coordinación ad hoc de cuatro o cinco personas en promedio, que pueden incluir personas exteriores al comité de redacción. Estos grupos se auto-organizan y deciden sus modos de funcionamiento, lo que permite respetar las disponibilidades de cada una y garantizar la diversidad de las contribuciones. La evaluación de los artículos y textos sometidos a la revista se realiza por el grupo coordinador de cada número, así como por dictaminadoras exteriores especializadas en el tema a evaluar.

La presencia de una importante cantidad de jóvenes feministas en el comité de redacción es un elemento importante en la dinámica de la revista. $N Q F$ desea abrir un espacio a las nuevas generaciones, en su construcción intelectual y a través de la publicación de artículos. De hecho, también con el propósito de contribuir a apoyar el desarrollo de los estudios feministas en la academia, NQF ofrece un apoyo especial a las investigadoras feministas que empiezan su trayectoria profesional.

Otra forma de abrir la revista lo más ampliamente posible, es a través de los llamados a contribución que ahora se sacan casi sistemáticamente para el dossier principal de cada número. Estos llamados a contribución intentan dirigirse tanto al medio académico, como a los colectivos militantes vinculados a las problemáticas que se quieren tratar en los dossieres. 
La revista se estructura alrededor de diferentes rúbricas fijas. El "gran ángulo" propone un dossier temático central. Por ejemplo, los tres números de 2002 abordaron sucesivamente las violencias contra las mujeres, la custodia parental y los "repertorios" de lo masculino. La rúbrica "campo libre" publica artículos que no se vinculan al tema específico del número, sirviendo especialmente para integrar textos que hayan sido propuestos "espontáneamente" a la revista. El "recorrido" está dedicado a entrevistas con militantes y/o mujeres con una trayectoria de especial interés, explicando su trayectoria feminista, analizando la historia de una experiencia, de una idea o de una teoría. Las "notas de lectura" pueden reseñar tanto libros, como coloquios o eventos militantes que nos interesa poner en perspectiva. Finalmente, la rúbrica "colectivos" nos permite dar la palabra a asociaciones, redes o grupos de investigación feministas que quieran presentar sus actividades y abrir un debate sobre los problemas que enfrentan.

La existencia, y sobre todo la formalización, de todas y cada una de estas rúbricas -que existían bajo una u otra forma en la fórmula anterior, pero menos formalmentedemuestra la importancia que queremos dar al desarrollo de la investigación feminista, a la vez que muestra nuestra voluntad de participar en la renovación del movimiento feminista, dando a conocer las experiencias de las mujeres comprometidas en las luchas cotidianas. Reflejo de estas luchas, espacio de debate teórico y político, herramienta de intercambio, la revista quiere sumarse al movimiento que hoy, con renovadas fuerzas militantes e intelectuales, apuesta en la erradicación del patriarcado.

\section{Un intento de balance teórico-político y práctico}

\section{1. ¿Legítima satisfacción?}

En apenas dos años, NQF ha conseguido llevar a cabo felizmente su proceso de transición: nueva imprenta y editorial, nuevo secretariado de redacción, nueva maqueta, redacción de la plataforma, creación de una sólida red de sustentación, consecución de nuevos financiamientos, y el sinfín de tareas administrativas consecutivas al cambio.

En medio de tantas tareas, $N Q F$ ha logrado respetar la nueva y ambiciosa periodicidad que se había propuesto (tres números al año). Ha realizado una importante campaña de difusión y promoción que ha permitido elevar a 350 el número de suscriptoras/es, cuya composición (aproximada) es la siguiente: 220 suscripciones individuales y 80 institucionales en Europa; fuera de Europa, 5 individuales y 15 institucionales; 35 suscripciones a precio reducido y 30 "solidarias" (se manda gratuitamente la revista a instituciones desprovistas de recursos). En cuanto a los países de residencia de las suscriptoras/es, Suiza arrasa, a raíz de la fuerte campaña de difusión allá realizada, con 180 suscripciones, seguida por Francia con 120. Existe un núcleo de suscriptoras en Canadá (20), mientras que los siguientes países sólo cuentan con menos de 10: España, Italia, Alemania, Gran Bretaña, Grecia, Portugal, Austria, Dinamarca, Finlandia, Países Bajos, Suecia, Hungría; y fuera de Europa, Australia, Japón y Brasil. En lo que a contenido se refiere, la revista ha ensanchado su repertorio. Si bien sigue claramente centrada en su perspectiva sociológica inicial, y a la vez en su interés de siempre por una visión pluridisciplinaria, el hecho que dentro del nuevo Comité estén representadas ahora casi todas las disciplinas de las ciencias sociales permite enriquecer bastante tanto las discusiones internas como el contenido de $N Q F$.

Tanto en su "viejo" período como en el actual, la revista ha publicado a bastantes investigadoras y/o militantes jóvenes y de alta calidad teórica-política, contribuyendo tanto a dar a conocer sus trabajos, como a aportar nuevas perspectivas al feminismo. También ha conseguido aportar informaciones y análisis provenientes del mundo militante. 
A pesar de sus pocos recursos, NQF ha podido presentar varias traducciones de artículos importantes provenientes del mundo anglófono e hispanófono, en su afán de dar a conocer al público francófono los trabajos feministas que se realizan en otras partes, en especial en el continente americano. Si bien se han realizado algunas traducciones de textos norteamericanos, el esfuerzo de la revista se ha dirigido especialmente hacía Latinoamérica y el Caribe, con la publicación de varios artículos, entrevistas y manifiestos de feministas mexicanas (en especial en torno a las mujeres -indígenas y no indígenaszapatistas), centroamericanas (Guatemala, El Salvador), bolivianas, dominicanas y brasileñas (con especial énfasis en la cuestión de las luchas de las mujeres negras en contra del racismo, del sexismo y del heterosexismo), destacando el número especial ya mencionado sobre feminismos latinoamericanos y caribeños, además de varios textos sobre el movimiento de mujeres, feminista y lésbico de la región. De hecho, el tema del lesbianismo y el análisis de la heterosexualidad como sistema, que pocas veces sale a la luz en las revistas académicas, incluso aún hoy, en las revistas feministas, a no ser que sean "especializadas" sobre el tema, también ha sido abordado repetidas veces, con textos franceses, españoles, latinoamericanos y caribeños.

\section{2. Puntos débiles}

Sin embargo, aún falta avanzar más en varios aspectos teniendo en cuenta que lo que pasa en $N Q F$ refleja la situación más global del feminismo europeo (debilitamiento, institucionalización, pérdida de radicalidad), de la academia (infra-representación de mujeres de origen extranjero y de clases populares), e incluso del clima socio-político actual (vuelta al conservadurismo, cruentos ataques hacía las mujeres y el feminismo, exacerbación del racismo).

Por un lado, la nueva composición del Comité de redacción, si bien refleja un avance importante, sigue dejando de fuera muchas feministas. Si bien hay militantes entre las universitarias que componen el Comité de redacción, es difícil motivar a mujeres puramente "militantes", quienes si no tienen a la vez un anclaje académico pueden hallar los debates y las preocupaciones del Comité demasiado "intelectuales". También hay muy pocas mujeres que no sean blancas-occidentales en el Comité, a pesar de que la presencia de inmigrantes en Europa es cada vez más importante, y que sus problemáticas y luchas son cada vez más centrales en las dinámicas socio-políticas de los diferentes países.

Esto a su vez se refleja en el contenido de la revista, que a veces puede considerarse de difícil lectura para las no-especialistas, o sea elitista. El (relativo) encerramiento en disciplinas estrechamente definidas, cuya "jerga" disciplinaria es un obstáculo para su apropiación por el conjunto de mujeres ( $y$ hombres) que podrían beneficiarse de estas reflexiones e intervenir en ellas, constituye una seria limitación -que no es propia de la revista pero que nos debe alertar, en cuanto feministas. Esto a su vez se agudiza con el precio de la revista. Si bien es cierto que existen suscripciones solidarias y una tarifa reducida, para muchas estudiantes, mujeres inmigrantes o desempleadas, $N Q F$ sigue siendo bastante cara. Obviamente, la revista no puede resolver ni asumir el conjunto de las problemáticas sociales, pero desde su posición feminista, militante, debe preocuparse por ampliar el círculo de sus lectoras y contribuidoras hacia todos los sectores, y no solamente consolidarse hacia el mundo académico, lo que implica no cejar en su empeño hacia las mujeres jóvenes, pobres, extranjeras, objeto de racismo, etc., quienes tienen mucho que aportar al feminismo, tanto teórico como práctico.

Finalmente, sería bueno que la revista prosiguiera y consolidara sus esfuerzos de traducción y de vinculación con otras revistas feministas, para ampliar las perspectivas teóricas y políticas del público lector francófono. En especial, es una pérdida considerable 
para nuestro desarrollo intelectual y práctico no conocer los trabajos de muchas feministas de los países del Sur -quienes en cambio conocen bastante el trabajo que se realiza en los países del Norte. Para avanzar en este sentido, hace falta dinero, y ciertamente no es tan fácil conseguirlo, pero también es necesaria sobre todo una decisión política firme basada en el convencimiento que el euro-etno-centrismo es un lastre. Por este camino vamos, pero aún hace falta mucho trecho. Y ciertamente, el financiamiento que se ha obtenido siempre es frágil. Aunque la revista esté en una posición mucho más sólida que en 1991, sigue vigente el llamado de Delphy: ihabrá que seguir luchando con ardor por los estudios y el movimiento feminista!

A modo de conclusión: los proyectos y las preguntas. Ciertamente, a sus 22 años de existencia, Nouvelles Questions Féministes se enorgullece del trabajo realizado, con altos y bajos pero con una férrea voluntad de seguir contribuyendo desde su posición teóricopolítica, no sólo al desarrollo y a la legitimación del feminismo académico, sino también a la construcción teórico-política del movimiento feminista, y sobre todo al afianzamiento y profundización de una perspectiva determinadamente radical, materialista y anti-esencialista. $N Q F$ se ha ganado una merecida fama de calidad teórica, de abridora de debates, a la vez que ha contribuido a crear y a demostrar la importancia del "feminismo materialista" francés $y$, de forma más amplia, de un feminismo radical que sigue apuntando a la destrucción del patriarcado, a pesar del retroceso ideológico actual en el que se debaten tanto el feminismo a escala mundial, como las demás teorías políticas progresistas. Aunque aún insuficientemente, ha logrado dar a conocer en Francia pensadoras y luchadoras importantes del resto del mundo (Andrea Dworkin, Adrienne Rich, Ximena Bedregal, las mujeres indígenas zapatistas, Ochy Curiel, Stevie Jackson, Mercedes Oliveira, Norma Mogrovejo). Ha ofrecido un espacio de difusión para el trabajo de varias investigadoras jóvenes y prometedoras -aunque tenga que trabajar bastante más hacia las mujeres provenientes de la emigración. Sigue intentando combinar teoría y militancia, feminismo con anticapitalismo y antiracismo, sin perder de vista la perspectiva lésbica radical y la crítica al sistema político de la heterosexualidad.

Aunque haya que seguir luchando sin descanso para conseguir financiamiento, la continuidad de la revista parece bastante bien garantizada con el nuevo equipo, el cual está pensando en ampliarse nuevamente para incorporar a más jóvenes feministas y militantes, cuyo interés por integrarse a $N Q F$ refleja, pensamos, la vitalidad del proyecto y el carácter acertado de sus orientaciones.

Terminaré con algunas preguntas antes de presentar nuestros proyectos futuros, con la esperanza de trabar alianzas y entablar o profundizar relaciones.

1. Hoy se están desarrollando bastante, en todo el mundo, los estudios "de género". A favor de este desarrollo, la noción "de género" corre el riesgo de perder cierto rigor intelectual -usándose cada vez más como un eufemismo para decir "mujeres", o para no decir "feminista". ¿Cuál puede ser el papel de nuestras revistas para evitar perder en profundidad teórico-política lo que ganamos en la extensión de nuestros conceptos?

2. La mundialización neoliberal ha aumentado tremendamente las desigualdades entre Norte y Sur, entre mujeres y hombres e, incluso, entre las mujeres (del Sur, inmigrantes, del Norte). Se ensancha la distancia entre las mujeres urbanas, con trabajo fijo y más o menos protegido (como somos las académicas), y las demás. A nivel de movimiento, existe una reflexión crítica importante, en especial en América Latina y el Caribe, sobre el divorcio entre las "expertas" y "consultoras" por un lado, y las militantes "de base" y mujeres de los sectores populares, negras, indígenas y pobres, por otro. Desde nuestras revistas y nuestra posición académica, ¿cómo podemos contribuir a revertir esta tendencia? Del movimiento feminista venimos, allí está nuestra fuerza y nuestra razón. ¿Cómo podemos 
hacer que nuestra producción teórica realmente refleje las problemáticas, necesidades e intereses de las mujeres en lucha? ¿Cómo podemos hacer que nuestros avances teóricos lleguen hasta todas las que los pueden utilizar y no sólo se quede en el mundo académico?

3. Se multiplican los campos en los que la perspectiva feminista y de género han incursionado. Aunque sea rica esta multiplicidad, talvez nos haga falta mayor discusión y coordinación para tener un panorama general de los avances teóricos del pensamiento feminista en su conjunto, y pensar en algunos temas centrales y candentes que nos convendría profundizar -cada una desde su inserción disciplinaria y geográfica. Los temas de la globalización neoliberal y la resistencia a la misma, de las crecientes desigualdades entre mujeres, del racismo y de la laicidad, versus el desarrollo de los integrismos, podrían constituir algunas pistas para hacer confluir nuestras investigaciones y reflexiones. Aprovechando la consolidación (relativa) de nuestros espacios en cada país y lugar, ino valdría la pena pensar en algunas orientaciones comunes para construir verdaderos conocimientos interdisciplinarios e internacionales sobre los temas que afectan cotidianamente a las mujeres en el mundo?

En cuanto a la propia $N Q F$, entre nuestros proyectos más inmediatos destacan una probable colaboración para un número especial con una revista canadiense de derecho; varios números especiales sobre temas como las transiciones post-comunistas, la interdisciplinaridad, la guerra, la salud y el trabajo. También se está planteando realizar un número especial sobre "Luchas radicales en América Latina y el Caribe", centrado en las luchas lésbicas, indígenas, negras, el feminismo autónomo, y una reflexión sobre las luchas armadas -entre otros. Este proyecto tiene una finalidad doble: dar a conocer directamente a las francófonas las luchas y los análisis de las protagonistas, mujeres militantes, latinas y caribeñas, a menudo no académicas, en vez de escribir "sobre" ellas (como académicas, Europeas, blancas), y simultáneamente, reunir y devolver para las latinoamericanas y caribeñas este conjunto de experiencias, por medio de una publicación en español. Ojalá encontremos entre ustedes algún eco para entablar colaboraciones en este sentido -iy en otros! Porque nuestra esperanza y convicción es que, más allá de los esfuerzos académicos y teóricos que cada una realizamos en nuestro lugar de residencia, el feminismo será militante y callejero -negro, indígena, árabe, asiático, africano, lésbico-, popular e internacional, io no será!

\section{Notas}

Copyright ( $\odot 2004$ by Revista Estudos Feministas.

' Christine DELPHY, 1991, p. 12.

${ }^{2}$ Françoise ARMENGAUD, 1991, p. 13

${ }^{3}$ ARMENGAUD, 1991, p. 14. 


\section{Fuentes}

DELPHY, Cristine. "Editorial”. Nouvelles Questions Féministes, Paris, n. 16-17-18, p. 1-12, 1991.

ARMENGAUD, Françoise. "Particularisme et universalisme". Nouvelles Questions Feministes, Paris, n. 16-17,18, p. 13-15, 1991.

Nouvelles Questions Féministes: 22 Years Deepening in a Radical, Materialist and AntiEssentialist Feminist Perspective.

Abstract: This article presents the main theoretical and political perspectives, as well as the herstory, of the oldest french-speaking scientific feminist review, Nouvelles Questions Féministes, which has been created 22 years ago by Simone de Beauvoir and others. One of the main distinctive point of this review, is its will to unite theoretical quality and an activist, political position, embedded in a very firm radical, materialist and anti-essentialist perspective. In the last 22 years, Nouvelles Questions Féministes published articles which have been critical for the feminist movement. In the midst of the "backlash 90ies", the review started facing hard times, with no institucional backing, and almost had to close down. But it started a new life in 2001 - with the same political perspective - , in the hands of a dynamic group based at the Lausanne University. Key-words: feminism, review, materialist, radical, anti-essentialist

74 Estudos Feministas, Florianópolis, 12(N.E.): 63-74, setembro-dezembro/2004 